\title{
Benign smooth muscle tumours of the oesophagus
}

\author{
N.R. B A R R E T T
}

There are two good reviews of this subject. The first, by Rose, described the cases reported up to 1936 ; the second, by Johnston, Clagett, and McDonald (1953), brought the matter up to 1952 and added a substantial series treated at the Mayo Clinic.

The fact that this topic has been reviewed by two such authorities might suggest that there is nothing useful left to say. But the vagaries of disease are such that the possibilities are never covered. If one considers such a common entity as acute appendicitis it is still certain that unique exigencies will crop up from time to time.

Here is a résumé of the knowledge available in papers about these tumours.

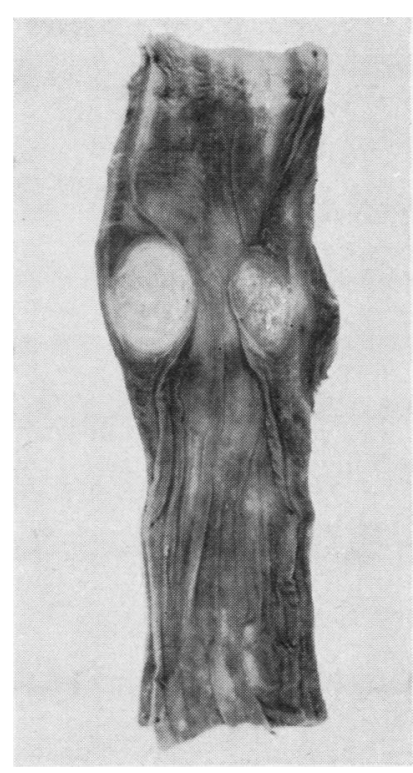

FIG. 1

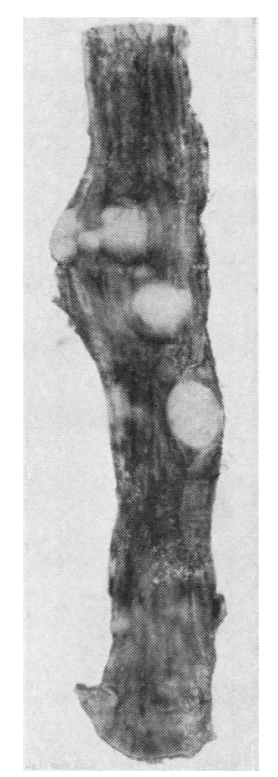

FIG. 2
FIG. 1. Salitary leiomyoma. Found at autopsy and symptomless during life.

FIG. 2. Multiple leiomyomata. Found at autopsy and symptomless during life.

(Both specimens are in the Pathological Museum of the Royal College of Surgeons of England.)
Tumours of smooth muscle arising in the oesophagus are said to be rare. There are less than 200 cases in the world literature. They occur more commonly in men than in women.

The onset of symptoms (or discovery of the lesion) generally occurs at an earlier age in leiomyomata (20 to 60 years) than it does in leiomyosarcomata (50 to 80 years). The former are symptomless in $50 \%$ of cases: they are discovered in the course of a routine check-up or as a result of a radiograph of the chest. If there are symptoms they are dysphagia or regurgitation, and these occur only if the tumour is large or if it encircles the gullet. Bleeding hardly ever occurs from leinmyomata; this is because the oesophagus is a transit tube in which food does not stagnate or digest.

By contrast leiomyosarcomata have a short history of symptoms that may include dysphagia, pain, bleeding, loss of weight, and obstruction. They simulate carcinoma of the gullet.

Physical examination is negative in simple and malignant varieties ; but it is claimed that a combination of radiology, oesophagoscopy, and biopsy yields the diagnosis in all cases nowadays. The overlying mucosa is said to be nearly always intact in benign tumours, however large they may be ; but the surface of malignant growths is often ulcerated. For this reason pre-operative diagnosis of leiomyosarcoma is difficult, and frequently it is not made until a thoracotomy has been done and histological preparations are available.

Leiomyomata vary in size from a millet seed to a large grapefruit. They may be sessile or pedunculated, solitary (Fig. 1) or multiple (Fig. 2), confined to the oesophagus or extending downwards across the cardia and into the stomach. They may bulge into the lumen of the gullet eccentrically or encircle the viscus and produce obstruction. They may protrude from the outer surface of the oesophagus into the mediastinum. Macroscopically leiomyomata look like grey, lobulated lipomata. They are firm to feel, like india-rubber, and they seldom break down. On section they look like a 'fibromyoma of the uterus'; they are multicentric and consist of whorls of smooth muscle surrounded by fibrous tissue that is not vascular. The colour of the cut surface is pink or white. By contrast leiomyosarcomata are yellowish-brown in 
colour, softer (because there are areas of necrosis) than simple tumours, and they are more adherent to adjacent structure. Neither variety has a capsule: some can be easily shelled out and others blend imperceptibly into the surrounding muscle.

The most satisfactory treatment of leiomyoma is enucleation, or oesophagotomy and resection of the tumour: leiomyosarcomata demand excision of the tumour together with the adjacent oesophagus, followed by reconstruction of the gap. The results of removal of leiomyomata are excellent, and the prognosis in malignant cases is not hopeless.

\section{MATERIAL}

During the years 1940 to 1963 I have seen eight patients suffering from leiomyoma and none who had malignant muscle tumours. The object of this report is to underline some features, gleaned from this experience, and to stress that diagnosis can be puzzling and treatment difficult.

CASE 1 This man aged 33 was the mate in a ship carrying grain from Australia. He noticed that every time they loaded wheat he suffered from 'severe asthma.' He had no physical signs or symptoms apart from this; but a radiograph showed a typical leiomyoma situated in the middle third of the oesophagus (Fig. 3). No abnormality, apart from lateral deviation

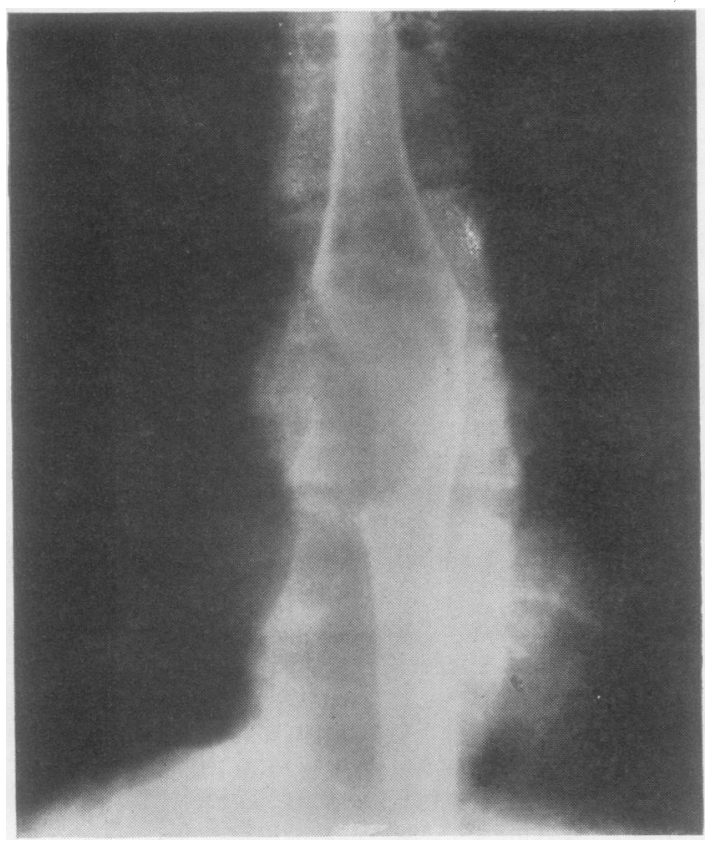

FIG. 3. Case 1. Radiograph of the tumour. of the gullet, was detected at oesophagoscopy. The $\stackrel{\overline{5}}{9}$ tumour, which was the size of a billiard ball and intra- $\overline{0}$ mural, was enucleated at thoracotomy. After this 으 operation he stated that his 'asthma' had been cured.

CASE 2 A man aged 59 was admitted with a clinical history, and bronchoscopic confirmation, of a car- ${ }^{-}$ cinoma in the right lower lobe bronchus. At operation $\overrightarrow{0}$ a leiomyoma the size of a small orange was palpated and removed from the lower part of the oesophagus: $\vec{\omega}$ the tumour in the lung was inoperable.

This case shows that leiomyomata may be dis- $\overrightarrow{\overrightarrow{0}}$ covered unexpectedly at thoracotomy done for $\dot{T}$ some other lesion. Alternatively, I have had two $\vec{\infty}$ patients, whom I diagnosed clinically and radio- $O$ logically as having leiomyomata, who had in fact got carcinomata, above which were enlarged $\overrightarrow{-}$ mediastinal glandular metastases. The latter had caused smooth indentations in the gullet that con-⿳亠丷厂 cealed the carcinomata lower down, and at oesophagoscopy these indentations resembled intramural leiomyomata.

CASE 3 A man aged 54 complained of mild, intermittent attacks of dysphagia. He had no signs or symptoms, but radiographs revealed a large filling defect in the middle third of the oesophagus (Fig. 4). $\mathbb{D}$ At oesophagoscopy a mobile, smooth, intraluminal tumour was seen. It was ulcerated over an area the size of a sixpence and seemed to be sessile and protruding into the lumen from a base that involved more than half the circumference of the gullet. Operation showed how wrong this opinion was, because the leiomyoma was pedunculated: the pedicle was no larger than a piece of thin string (Fig. 5). How the blood supply had been maintained through such a $\frac{0}{0}$ slender pedicle is difficult to understand. Histologically this tumour was reported upon as a typical leiomyoma by some, as a leiomyoma containing nerve elements by others, and also as a neurofibroma. Macro-

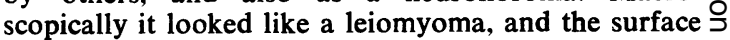
was smooth and not furrowed like that seen in $D$ Figure 18.

CASE 4 A young man was successfully operated upon for a perforated gastric ulcer. Subsequent investiga- $N$ tions revealed a large, homogeneous, lobulated opacity in the posterior mediastinum. A barium swallow 0 showed the gullet to be displaced, and flattened like a tape, around the mass (Fig. 6); a diagnosis of mediastinal lipoma or fibroma was made. At opera- $\cong$ tion the tumour was found to be an oesophageal ${ }_{-}^{+}$ leiomyoma that extended from the lower oesophagus $\frac{T}{3}$ onto the lesser curve of the stomach. It was success- $\frac{}{8}$ fully enucleated (Fig. 7), and the distended hiatus $\stackrel{\complement}{\complement}$ was repaired. This tumour resembled the large sub- $\mathbb{\mathbb { Q }}$ serous leiomyomata that sometimes arise from the serous surface of the stomach. 

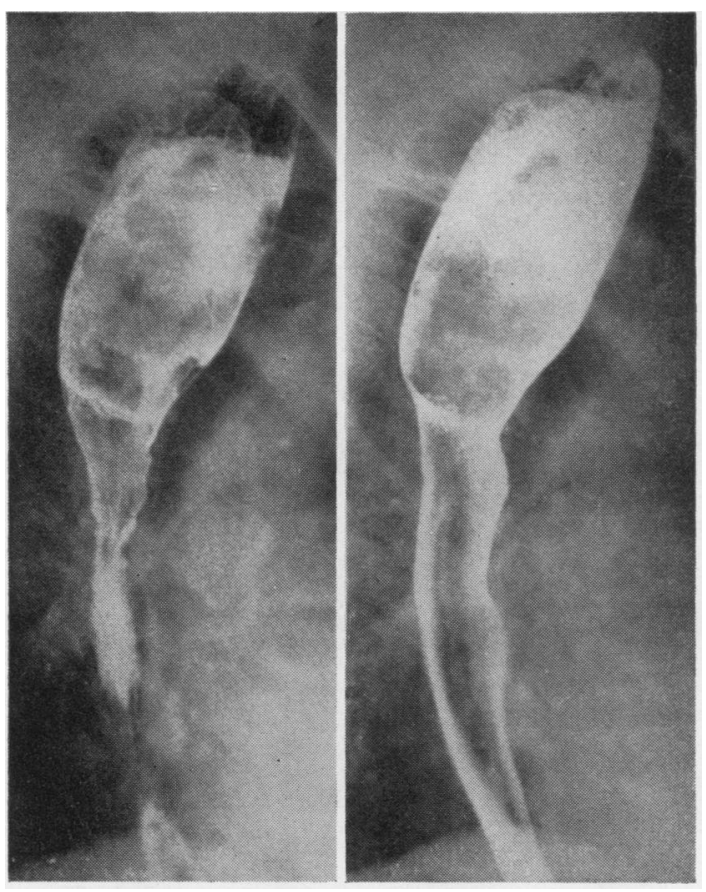

FIG. 4. Case 3. Radiograph showing the enlargement of the oesophagus caused by the pedunculated leiomyoma in the lumen.

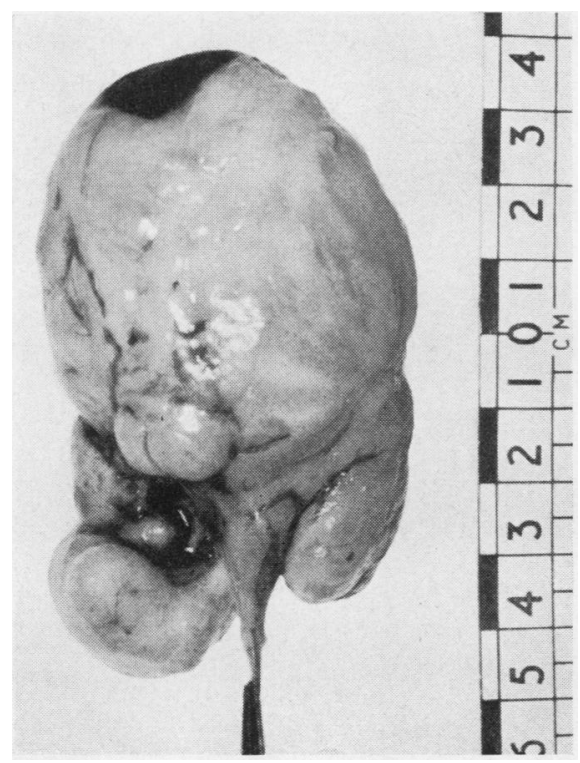

FIG. 5. Case 3. Note the small area of ulceration at the summit of the tumour and the slender pedicle at the bottom.

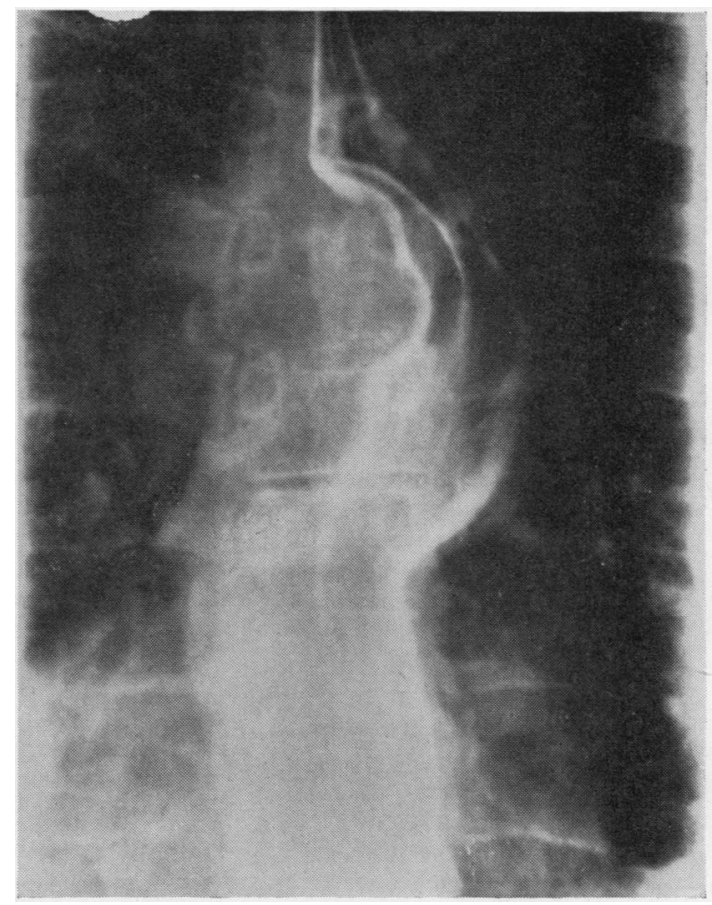

FIG. 6. Case 4. Radiograph showing the gullet displaced around the tumour.

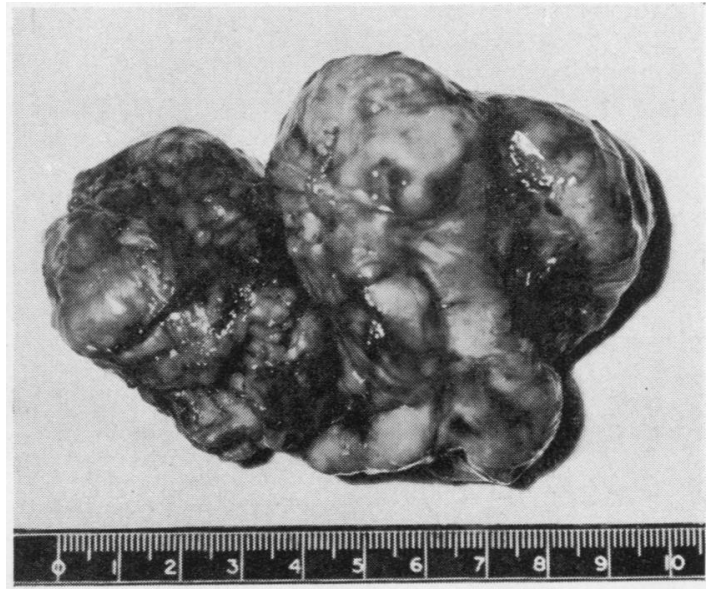

FG. 7. The tumour removed from case 4. 
CASE 5 A woman aged 28 was admitted complaining of prolonged and severe dysphagia. She was suffering from malnutrition and serious episodes of 'pneumonitis' due to repeated spill-over from the obstructed oesophagus. Radiographs revealed a long and tortuous oesophageal stricture affecting the middle third of the gullet. She died of 'intractable pneumonia' before anything surgical could be attempted. At autopsy a diffuse multicentric, lobulated, intramural leiomyomatosis of the oesophagus was found. There was no evidence of malignancy, although the lesion (which was $3 \frac{1}{2}$ in. $(9 \mathrm{~cm}$.) long) looked like a leather-bottle stomach. The specimen was mislaid during the war ; no other abnormalities were found.

CASE 6 A woman aged 60 was admitted with longstanding, severe dysphagia, vomiting after meals, and loss of weight. Investigations showed a calcified mass involving and obstructing the lower part of the oesophagus (Fig. 8). This was a calcifying leiomyoma (Fig. 9), and it shelled out without difficulty at thoracotomy. At the time of this operation the lower oesophagus (apart from the tumour) looked normal, but four years later this patient came back complaining of increasing dysphagia and retrosternal pain. She then had gross oesophagitis, at $25 \mathrm{~cm}$. from the incisor teeth, a moderate stricture at this level, a lower oesophagus lined by columnar epithelium below the

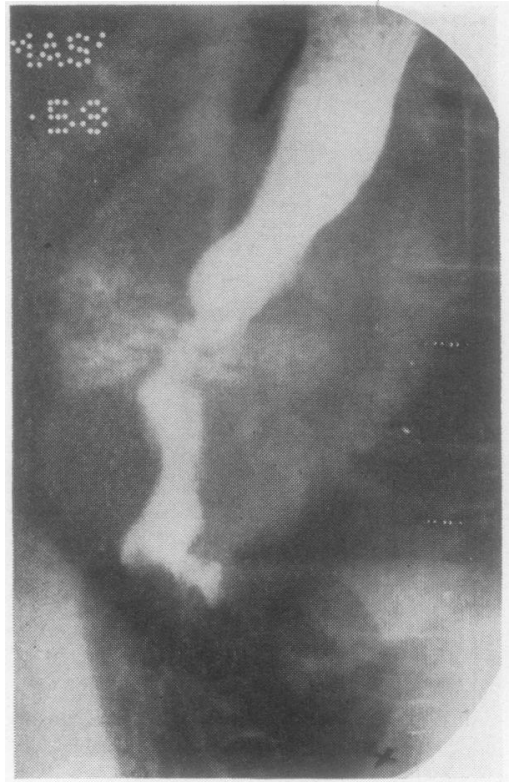

FIG. 9. Case 6. Another radiograph showing the calcification in the tumour.

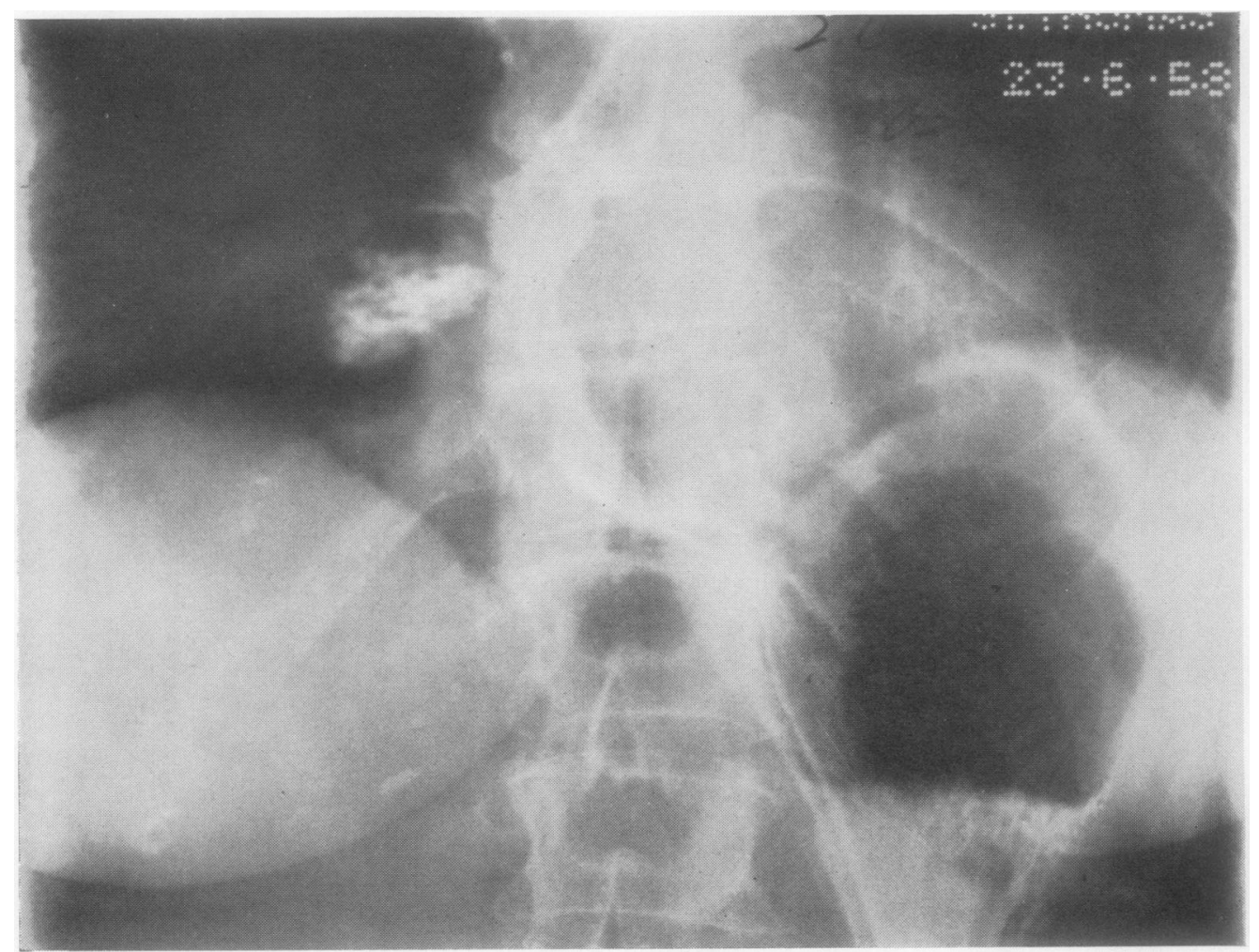

FIG. 8. Case 6. Radiograph showing the calcification in the leiomyoma and the distortion it had produced in the lumen of the lower oesophagus. 


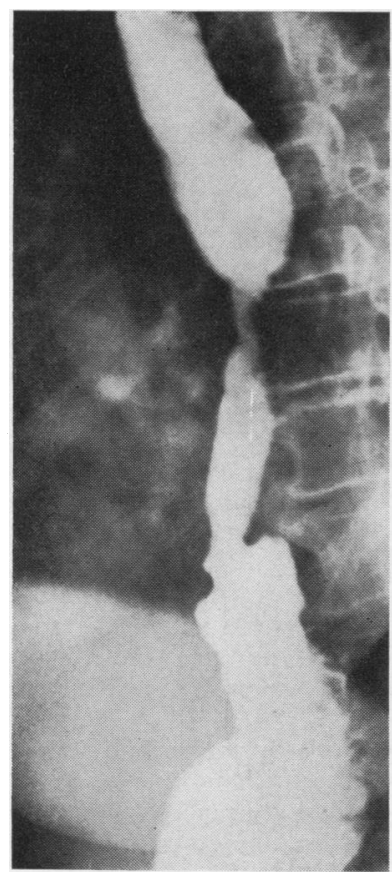

FIG. 10. Case 6. This radiograph of a barium swallow was taken four years after removal of the calcified leiomyoma. It shows an oesophageal stricture, a lower oesophagus lined by columnar epithelium, and a sliding hiatus hernia.

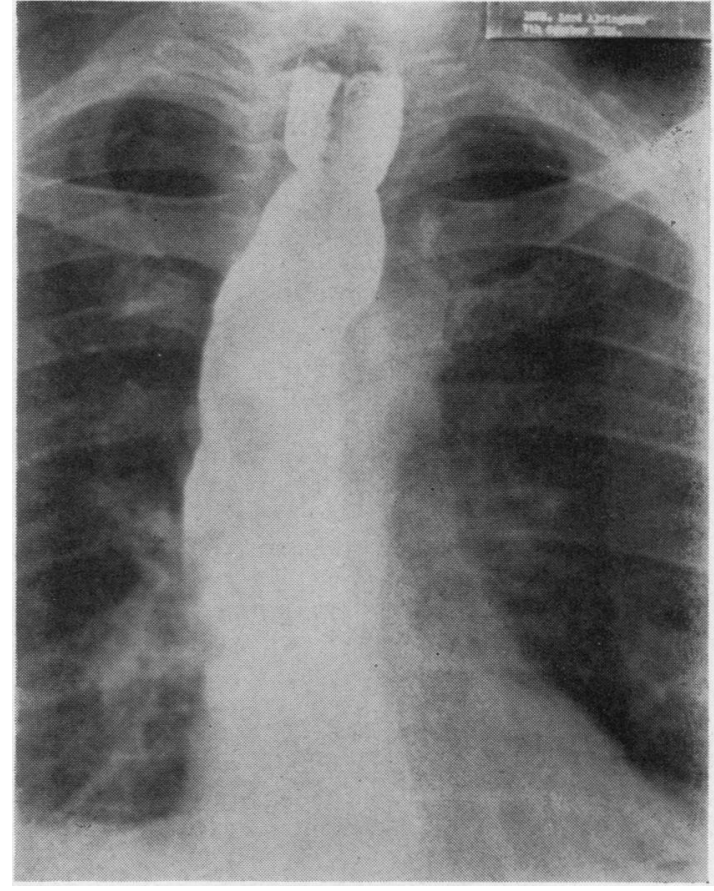

FIG. 12. Case 8. Radiograph taken in 1938. A barium swallow showing achalasia of the cardia and, possibly, $a$ filling defect in the middle third of oesophagus.

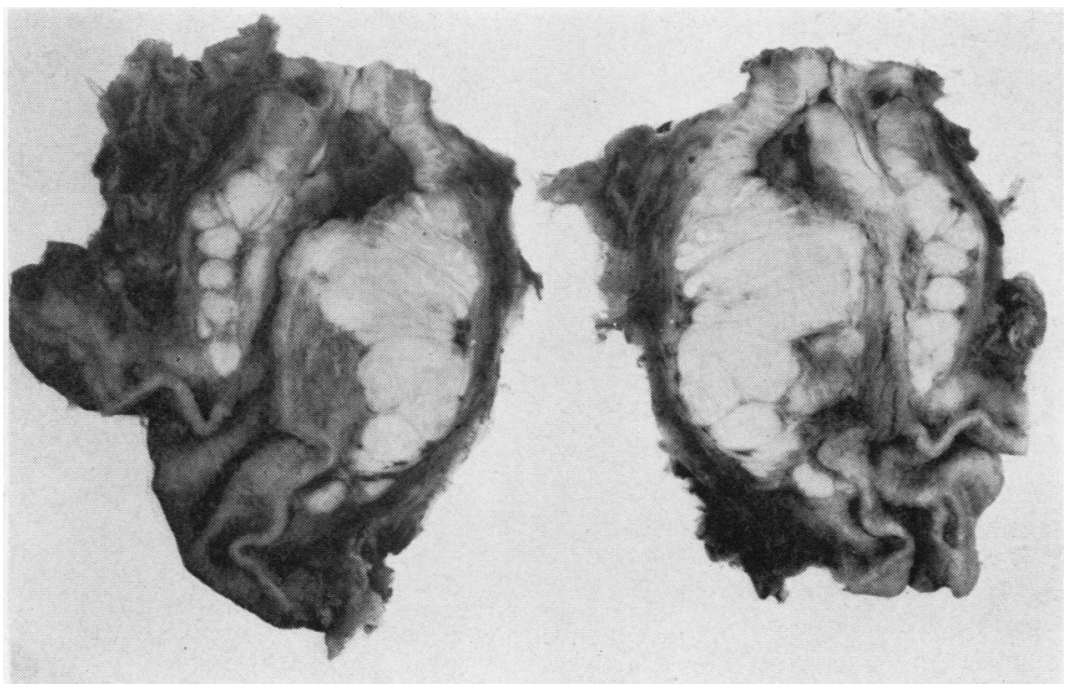

FIG. 11. Case 7. The cut surfaces of the specimen removed at operation. The timour encircles the lumen of the lower oesophagus and was mistaken for a carcinoma radiologically and at operation. 
stricture, and a sliding hiatal hernia below this (Fig. 10). Her symptoms were relieved by surgical reduction of the hiatus hernia, and at this operation no recurrence of the leiomyoma was found.

CASE 7 A woman aged 81 complained of increasing dysphagia of one year's duration. She had no physical signs but radiographs revealed a persistent stricture in the lower oesophagus that was tortuous, irregular, and exactly like a carcinoma. At oesophagoscopy a firm stricture was found at $35 \mathrm{~cm}$. from the incisor teeth and this was thought to be the top surface of a carcinoma ; the possibility of leiomyoma had not been seriously entertained. A biopsy was taken and this revealed 'normal squamous epithelium, submucosa, and a few muscle fibres.' A thoracotomy was done and she proved to have a leiomyoma that was multicentric and that encircled the gullet; as the correct diagnosis was not made (even at operation) the lower oesophagus containing the tumour was resected (Fig. 11): she has remained well.

CASE 8 The patient, a middle-aged man, was well until 1938, when he noticed intermittent dysphagia. A barium swallow confirmed the diagnosis of achalasia of the cardia (Fig. 12), and there is no doubt that this was correct. He was treated by bouginage but rebelled against this 'barbarous piece of circus-craft.' The dysphagia became so severe that a gastrostomy was done and he was fed in this way for two years. Throughout the war he lived on milk alone, and he settled down to a life of invalidism. During these years he developed gross hypertrophic pulmonary osteoarthropathy with pains in the hands and feet and effusions into the knee-joints. He retired to bed and thereafter became bedridden. The diagnosis made, and the treatments prescribed for his condition, were interesting, inaccurate, and various. He lost a great deal of weight. Eleven years later radiographs showed an enormous intrathoracic opacity that looked more like a mediastinal tumour than achalasia (Fig. 13). Barium studies were indefinite and not understood: the emulsion appeared to be fragmented by the opacity as though there were a number of false oesophageal channels.

By this time the patient was seriously ill. and Heller's operation was successfully done but without ameliorating the symptoms. He complained of dysphagia and an irrepressible cough whenever he swallowed.

Because Heller's operation had failed to relieve his symptoms and because nothing unusual had been felt in the lower oesophagus at this operation it was decided that oesophagoscopy was now essential. This

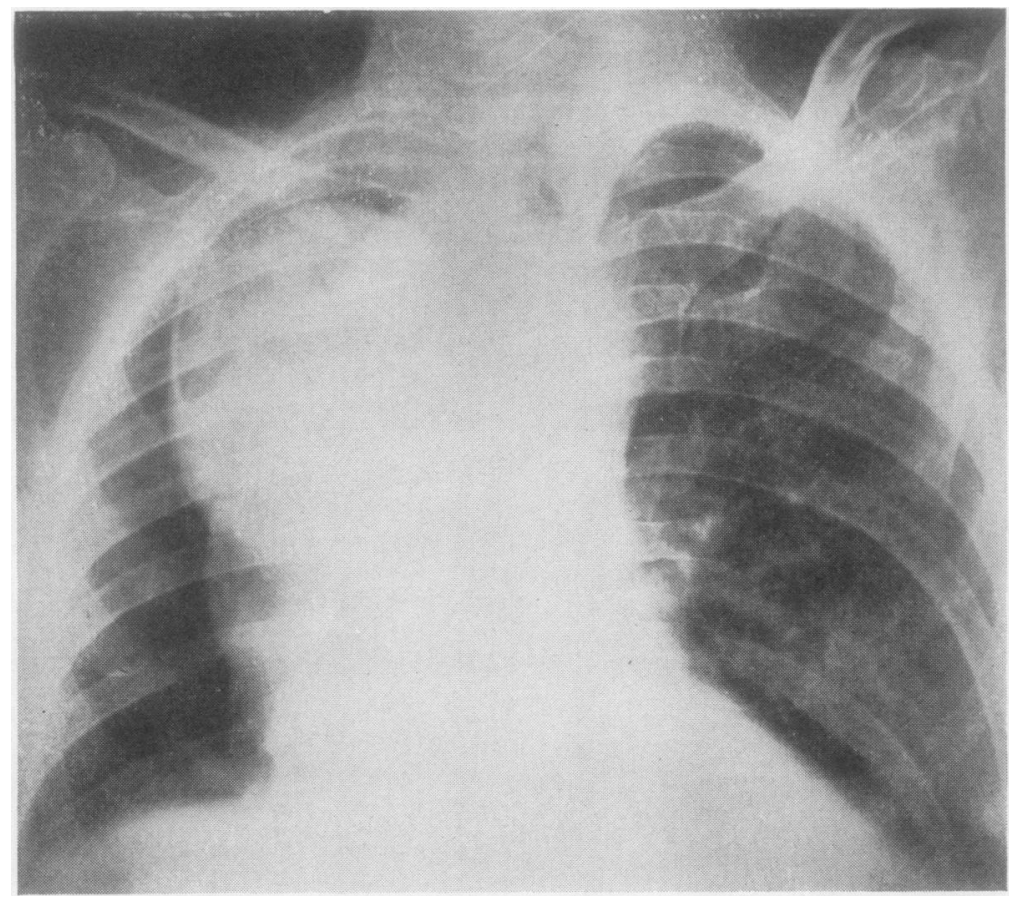

FIG. 13. Case 8. The radiological appearances in 1949. The opacity in the mediastinum looked more like a tumour than achalasia of the cardia, but, because dysphagia was serious and because the original diagnosis had been achalasia, an Heller's operation was done without relief of the symptoms. 
was done and he died without recovering consciousness. The oesophagoscopist was a surgeon, most experienced in this investigation. Here is his report and it is indefinite: 'The upper end of the oesophagus is lined by normal, pale epithelium; but at $20 \mathrm{~cm}$. the mucous membrane is thickened and bright red. Its surface is covered by a milk-like film in patches (this was in fact milk), there is no enlargement of the lumen, and the whole organ seems to be displaced forwards and to the right in a "bumpy" fashion. There is no retained food, but there is some fluid in the gullet that might be pus: but no orifice was found through which this might have exuded. The lumen was extremely difficult to follow-this was partly because it was encroached upon by a series of large, smooth "bumps" that were extra-mucosal. As the oesophagoscope was passed down over a stillette the "bumps" were felt to be firm but not hard. The appearances from $15 \mathrm{~cm}$. downwards might possibly be due to a leiomyomatous tumour-there was no obstruction at the cardia.'

At autopsy the patient had a gross megaoesophagus, but in addition there were two leiomyomata of the oesophagus. One was enormous (and in retrospect was

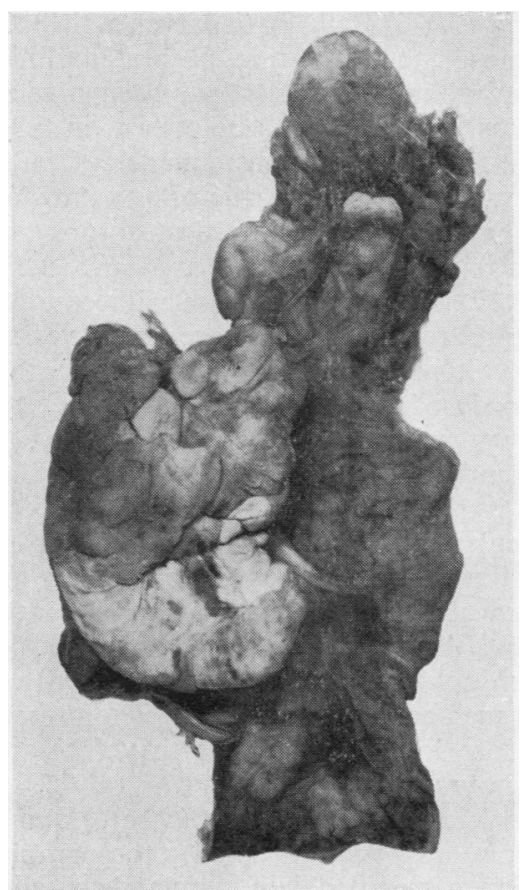

FIG. 14. Case 8. Autopsy specimen showing the tongue and the upper two-thirds of the oesophagus. There is gross dilatation of the gullet due to achalasia of the cardia. In addition there is a giant leiomyoma, arising from the middle third of the gullet, and a smaller one just below the cricopharyngeal sphincter. (The specimen is in the Pathological Museum of the Royal College of Surgeons of England.) discernible as a small tumour in the original film that had been diagnosed as achalasia 18 years previously); it was lying in the upper half of the right chest, and the wall of the gullet was tightly stretched over it (Fig. 14). The other was about the size of a small orange: it lay just below the crico-pharyngeus muscle sphincter and accounted for the fact that the patient 'spluttered' every time he tried to swallow. There was no evidence of malignancy; both tumours were leiomyomata.

These tumours were large and the gullet was so distorted and stretched that successful excision would have been difficult: but if a correct diagnosis had been made this patient might have been relieved, even after all these years of faulty diagnosis and ineffective treatment.

\section{INCIDENCE}

These tumours are not as rare as the published cases suggest. Every thoracic surgeon has experience of one or more that have never been published: and those who are interested in oesophageal work do not regard leiomyomata as exceptional. The fact that pathologists and morbid anatomists do not find them at autopsy could be due to inadequate examination of the gullet.

\section{ONSET OF SYMPTOMS}

A leiomyoma growing in the wall of the oesophagus causes no symptoms for a long time. The fact that it can be present for many years is underlined by cases 6 and 8 described above (Fig. 15).

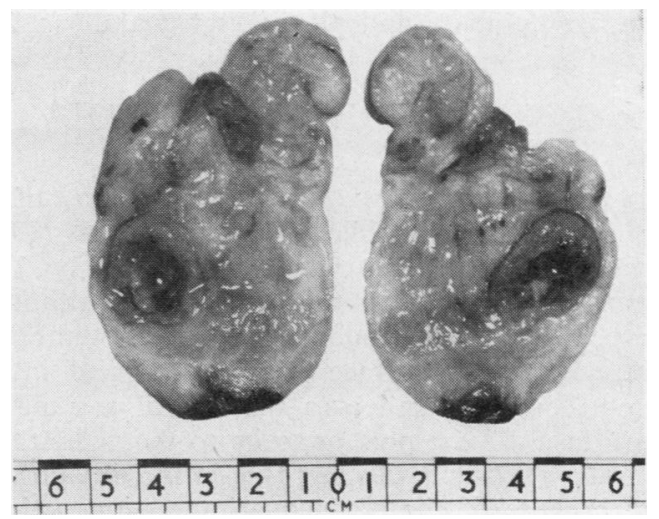

FIG. 15. The macroscopic appearances of a typical leiomyoma after enucleation.

Leiomyomata that encroach upon the lumen of the gullet are often silent because, although the area of circumference of the gullet from which they arise may be considerable, the remainder of the circumference is lengthened and stretched over 
the mass. Thus the lumen is crescentic and slit-like, and it is larger than normal. But if the tumour eventually encircles the gullet, like a collar, the patient experiences severe dysphagia, and is likely, upon clinical, radiological, and endoscopic evidence, to be diagnosed as having a carcinoma.

Tumours that arise from the muscularis mucosae, from the circular muscle, or from the smooth muscle of oesophageal blood vessels protrude into the lumen and are likely to become pedunculated because of the constant downward urge of food and peristalsis. Cases have been recorded in which leiomyomata, on long stalks and arising from the upper part of the oesophagus, appeared and disappeared suddenly and unaccountably in the mouth. Clagett showed such a patient at a meeting of the Thoracic Society in 1953, and there are historical records of great interest concerning tumours of this type (Monro, 1811 ; Mackenzie, 1884 ; Minski, 1895). It is stated that they do not prolapse into the stomach when situated in the lower part of the oesophagus.

If a leiomyoma is situated immediately below the cricopharyngeal sphincter (as was the upper of the two found in case 8 above) the patient complains of dysphagia and an intractable cough that occurs as soon as he tries to swallow.

Giant leiomyomata not only obstruct the oesophagus but encroach upon the space available in the mediastinum and pleural cavities. They may cause not only dysphagia but atelectasis, and they simulate lipomata and pleural fibromata.

\section{CONCERNING THE TUMOURS THEMSELVES}

PEDUNCULATED LEIOMYOMATA These are often large, elongated, mobile, and symptomless. The tumour reported here (case 3) measured 8 by 5 by $4 \mathrm{~cm}$. and on section it was not necrotic although the pedicle was so slender. The mucous membrane that covered its surface was normal and intact except for one small area the size of a sixpence (see Fig. 5) at the pole opposite to the pedicle. At operation it was easily palpable in the oesophagus but, because of the erroneous impressions gained at oesophagoscopy, it was thought to be intramural, and an incision was made over it with a view to enucleation. This incision opened the lumen of the oesophagus, rather unexpectedly, and it was only then that the true state of affairs was discovered. The tumour was intraluminal and pedunculated; these details were not easy to demonstrate before operation.
The fact that pedunculated leiomyomata are $\stackrel{\overrightarrow{\vec{P}}}{\stackrel{\overrightarrow{3}}{+}}$ mobile inside the gullet, and that they can range? up and down over a distance of several inches, 음 could be of use to the radiologist. When a circum- $-\frac{\overline{ }}{-}$ scribed zone of decreased density is picked up $\propto$ during a barium swallow, the patient should be radiographed again in the head-down position, and $\vec{\theta}$ the tumour may be found to have moved in the lumen. This information would be helpful to $\mathrm{a} \overrightarrow{\vec{\omega}}$ surgeon pre-operatively because, in these circumstances, an oesophagotomy will be necessary.

INTRAMURAL TUMOURS These are of three types. $\overrightarrow{0}$ The mass may be solitary and grow eccentrically? in the muscle of the oesophagus (case 1); this iso $\vec{\sigma}$ the usual finding. A tumour of this type convertso the lumen into a crescent. The tumour may sur- $]$ round the gullet, like a collar round a horse's neck $\vec{z}$ (cases 6 and 7); or it may be elongated and involve a long segment of the oesophagus (case 5). The symptoms vary accordingly.

The pathological findings in case 5 are unusual. They simulate the extraordinary tumour described ${ }^{\oplus}$ by Hall in 1916. Hall's patient was a girl who died, undiagnosed, of starvation. Repeated clinical and radiological investigations had been made, and a large mass was known to be present in the media- $\stackrel{0}{0}$ stinum. The radiographs, made after barium $\stackrel{\mathbb{Q}}{2}$ swallow, were not diagnostic of any known condi- $\overrightarrow{\vec{O}}$ tion. The gullet was removed at autopsy, and the 3 photographs of the specimen are reproduced (Figs. 16 and 17). Compare this case with case 5.

It appears that there are two varieties of ' muscle ' hypertrophy of the oesophagus that have been confused. On the one hand, there are cases, $\stackrel{\sim}{x}$ presenting with pain due to overactive muscle $\dot{\sigma}$ movements (and called 'acute spasm'), that have diffuse hypertrophy-or increase in thickness-of $\delta$ the circular muscle of the lower two-thirds of the oesophagus. These patients are possibly cases of $\frac{9}{7}$ early achalasia in which peristalsis has not yet $\supset$ been lost. At operation the thick muscle does not feel or look tumorous. By contrast, there are N others, such as Hall's patient, in whom the muscle masses are lobulated so that they feel and look $N$ like tumours.

Some leiomyomata calcify (case 6) and can be seen on radiographs and tomography. At operation a calcified leiomyoma feels, and looks $\frac{}{\varnothing}$ like, a large, lobulated, hen's egg, The tumour found $\stackrel{?}{+}$ in case 6 shelled out easily, and the mucosa was $\square$ not opened. Before mobilization it lay against the $\vec{O}$ descending aorta, and this was deviated from its normal position as it passed around the tumour. $\mathbb{D}$ The possibility of calcification in an aneurysm of the descending aorta has been considered. 


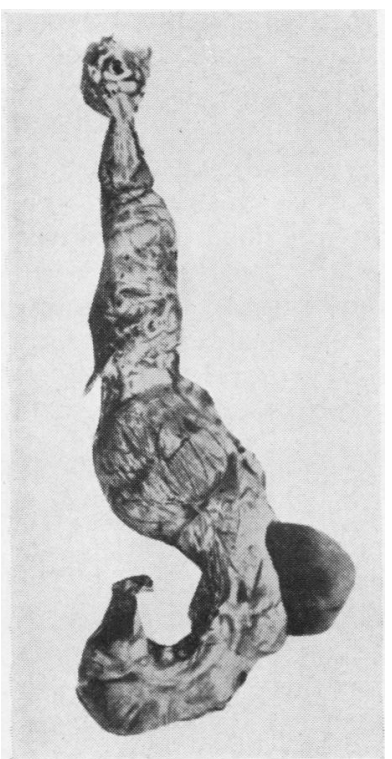

FIG. 16

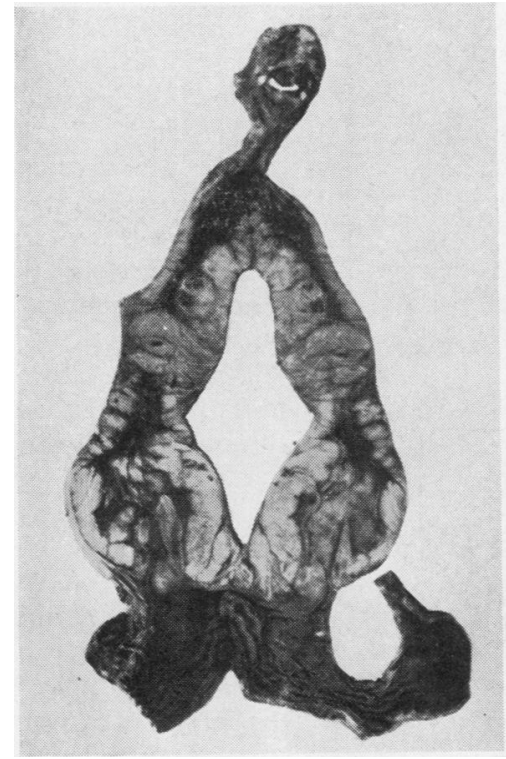

FIG. 16. This photograph, and that labelled Fig. 17, are reproduced from the paper by Hall (1916). The larynx is at the top of the specimen, and the stomach at the bottom. Note the enormous enlargement and distortion of the gullet.

FIG. 17. Hall's case (1916). The specimen has been opened, and the leiomyomatous process can be seen to involve the oesophagus from top to bottom. Case 5 in the present series was of this type but of much more limited extent.
ASSOCIATED CONDITIONS The following associated conditions have been described: diverticulum, achalasia of the cardia, hiatus hernia, malignant melanoma, and carcinoma. When any of these occur together with a leiomyoma the clinical picture and findings are obscure, and the presence of a benign muscle tumour may be missed.

\section{GENESIS}

Some surgeons have considered leiomyomata of the oesophagus to be congenital lesions that resemble hamartomata. But there is no evidence for this, and no cases have been reported in infants.

They have been found in the circular and the longitudinal muscle coats; and they may also arise outside these layers, from the muscularis mucosae or from the smooth muscle in the walls of the oesophageal arteries.

Leiomyomata may contain ganglion cells and fragments of nerve. Thus histological sections may not be sufficiently clear-cut to distinguish between leiomyoma and neurofibroma (case 3) (Fig. 18). In practice, the difference between these two tumours is not important to the surgeon: both should be removed, and, if removal is complete, neither recur.

Another confusion has arisen as a result of the haphazard use of the word 'polyp.' A polyp is a pedunculated 'tumour,' and oesophageal polypi may be leiomyomatous, neurofibromatous (or

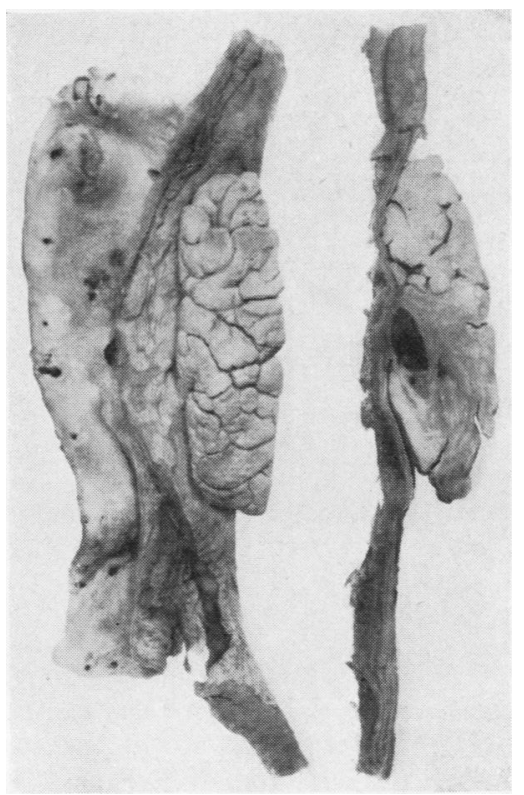

FIG. 18. This specimen is in the pathological museum of the Royal College of Surgeons of England. It shows some of the difficulties of diagnosis in these cases. The tumour in the gullet is a neurofibroma; but the cut surface (on the right) shows that it includes a small malignant melanoma (Dr. Prodger). 


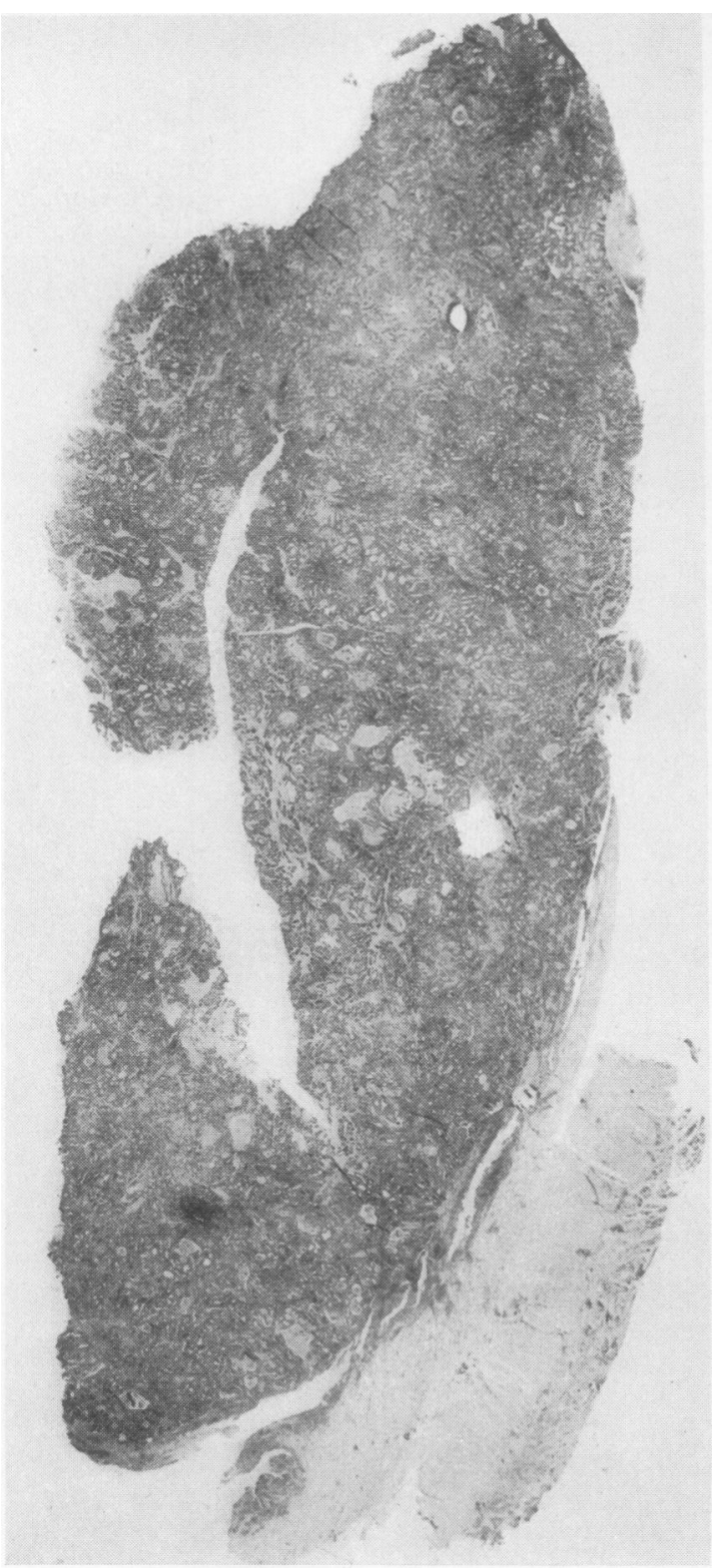

FIG. 19. This tumour was removed, by the late Mr. Gordon Cruickshank, from the lower third of the oesophagus. At operation he considered it to be a leiomyoma, but histologically it is an adenomatous polyp.

mixed), adenomatous, and malignant. To the operating surgeon there is no way of distinguishing these three simple varieties but they are different histologically. The late Mr. Gordon Cruickshank, of Leicester, sent me the clinical and operation findings (Fig. 19) of a man who had dysphagia caused by a large adenomatous polyp in the lower third of the oesophagus. He removed the tumour without difficulty, and at the time he considered it to be a leiomyoma. Histologically, the epithelium that composed the mass was 'gastric' in type, and the lower oesophagus was lined by columnar epithelium. It is worth while stressing that when a biopsy of such a 'tumour' is made at endoscopy only a small fragment is obtained, and this only tells a part of the story. My experience of diagnosis from endoscopy specimens taken from these tumours is disappointing.

Leiomyomata occur at all levels in the oesophagus; this has been challenged by some who believe that the upper third of the gullet is supposed to be coated by striated muscle. The arrangement of the muscle in the upper third of the oesophagus is a matter about which textbooks of anatomy differ. Whereas there is no doubt that the crico-pharyngeus sphincter is a striated muscle, there is dispute about where the change over to non-striated muscle in the oesophagus occurs. However this may be, the oesophagus functions as a physiological whole, and typical leiomyomata can arise at any level.

Small leiomyomata are unlikely to be discovered unless the oesophagus is properly examined at autopsy. Tumours the size of a hen's egg usually have typical radiological and endoscopic appearances. But it is the giant, lobulated masses that are often misdiagnosed, and this matters because, in spite of their size, many can be cured by surgical excision if the operation can be planned in advance.

An object in writing this paper has been to underline the fact that no individual surgeon has enough experience of these tumours to be dogmatic in his opinions. But the accumulated experience in the literature shows not only the variety of type and clinical effects but that a hopeful outcome can be expected if the condition is diagnosed correctly and early.

\section{REFERENCES}

IIall, A. J. (1916). A case of diffuse fibromyoma of the oesophagus, causing dysphagia and death. Quart. J. Med. 8, 409.

Johnston, J. B., Clagett, O. T., and McDonald, J. R. (1953). Smoothmuscle tumours of the oesophagus. Thorax, 8,251 .

Mackenzie, M. (1884). A Manual of Diseases of the Throat and Nose. Vol. II, p. 98. Churchill, London.

Mincl. Th. Polypen und polypenähnlichen Gewächse der Rachens und der Polypen und polypenähnlichen Gewachse der Rachens und der

Monro, A. (1811). The Morbid Anatomy of the Human Gullet, Stomach, and Intestines, p. 186. Constable, Edinburgh.

Rose, J. D. (1936). Myomata of the oesophagus. Brit. J. Surg., 24, 只 297.

Tanner, N. C. and Smithers, D. W. (1961). Tumours of the Oesophagus, p. 109. Livingstone, London. 